\title{
LA TELEVISIÓN IP (IPTV) Y LA TRANSMISIÓN MEDIANTE VDSL: REALIDAD Y PERSPECTIVAS DE NEGOCIO
}

\section{IP TELEVISION (IPTV) AND AND THE TRANSMISSION BY MEANS OF VDSL: REALITY AND PERSPECTIVES OF BUSINESS}

Joan Francesc Fondevila Gascón: Profesor de la Universitat Abat Oliba (UAO), Universitat Autònoma de Barcelona (UAB) y Universitat Oberta de Catalunya (UOC). Director del Centro de Estudios sobre el Cable (CECABLE) jffondevila@ilimit.es

\section{CURRÍCULUM VITAE}

Doctor en Periodismo por la Universitat Autònoma de Barcelona (España), Licenciado en Ciencias de la Información, Licenciado en Ciencias Políticas y Sociología, Máster en Periodismo y Ciencias de la Comunicación y Máster en Comunicación y Deporte. Profesor en la Universitat Abat Oliba CEU de Barcelona (España) de Barcelona, profesor del Doctorado en Sociedad de la Información y el Conocimiento y del Máster Universitario de Sociedad de la Información y el Conocimiento de la Universitat Oberta de Catalunya (España), y profesor de la Universitat Autònoma de Barcelona y la Universitat Politècnica de Catalunya (España). Es fundador y director del Centro de Estudios sobre el Cable (CECABLE)

\section{RESUMEN}

La introducción comercial de la tecnología VDSL puede dinamizar las soluciones de televisión IP (IPTV) y el periodismo audiovisual en general a través de la red de 
banda ancha. El análisis de las incipientes experiencias de televisión a través de VDSL en diversos países permite establecer una prospectiva que afecta a las diversas aplicaciones audiovisuales. El servicio de televisión adquiere así protagonismo ante los de telefonía e Internet dentro de los servicios de triple play, y puede combinarse en movilidad con el quadruple play.

\title{
PALABRAS CLAVE
}

Televisión - Periodismo Audiovisual - Tecnología VDSL - Banda Ancha - Soluciones IPTV

\begin{abstract}
The commercial introduction of the technology VDSL can stir into action IP television (IPTV) solutions and the audio-visual journalism in general across the broadband network. The analysis of the incipient experiences of television across VDSL in diverse countries allows to establish a futurology that concerns the diverse audiovisual applications. The service of television acquires protagonism like that before those of telephony and Internet inside the services of triple play, and can combine in mobility with the quadruple play.
\end{abstract}

\section{KEY WORDS}

Television - Audiovisual Journalism - VDSL technology - Broadband - IPTV Solutions 


\section{ÍNDICE}

1. La televisión de banda ancha (IPTV), expectante ante el VDSL

2. Aplicaciones prácticas de la televisión mediante VDSL

3. Conclusiones

4. Bibliografía

5. Notas

TEXTO

\section{La televisión de banda ancha (IPTV), expectante ante el VDSL}

La televisión sobre el IP (Internet Protocol), la IPTV, es una realidad incipiente en España y en otros países del mundo que absorbe el suficiente caudal de banda ancha como para que la infraestructura telecomunicativa sea suficientemente madura. La introducción de la tecnología VDSL (Very high bit-rate Digital Subscriber Line) puede relanzar las soluciones de IPTV y el audiovisual a través de la red de banda ancha. Las aplicaciones del periodismo audiovisual en IPTV, sea HDTV (Televisión de alta definición) o estándar (Delgado y Fernández Quijada, 2007), consumen ancho de banda a raudales. Además, desde una perspectiva teórica-descriptiva, de análisis deductivo, y de tipo metodológico cuantitativo y cualitativo, los usos de tecnología en el hogar acostumbran a estar interrelacionados entre ellos (CMT, 2006), de forma que lo habitual es disponer del servicio de televisión sumado a de juegos online, voz sobre IP (VoIP) y otros (véase Tabla 1). 
Tabla 1. Requisitos de ancho de banda para servicios
Aplicaciones
Downstream
Upstream
IPTV
$18 \mathrm{Mbps}$
$1 \mathrm{Mbps}$
Juegos online
$2 \mathrm{Mbps}$
$2 \mathrm{Mbps}$
VoIP
0,3 Mbps
0,3 Mbps
Internet
$10 \mathrm{Mbps}$
$4 \mathrm{Mbps}$
Videoconferencia
$2 \mathrm{Mbps}$
$2 \mathrm{Mbps}$
Total
32,3 Mbps
9,3 Mbps

Fuente: Elaboración propia a partir de datos de CECABLE y de ZyXEL (2008).

El proceso de convergencia entre audiovisual y telecomunicaciones, pese a ciertas divergencias en sus inicios (Garnham, 1996) y la plétora de exégesis que implica (Castells, 1995 y 1997; Pestano, 2001; Becerra, 2003; Ritzer, 2007), está cada vez más asentado. El fenómeno de la digitalización permite la reutilización y amortización de contenidos, para satisfacción del periodismo del siglo XXI. Los contenidos audiovisuales también se aprovechan de ello, incluso de forma más suculenta gracias a la consolidación del concepto de televisión de pago (Smellie, 1995). El hecho que las ofertas de triple play (televisión + telefonía + Internet) y quadruple play (añadiendo la movilidad) (ver Nota1) conlleven, aunque en ocasiones se ofrezca una opción 
televisiva gratuita, abonarse al operador, genera una dinámica estable de pago por uso y paso por ver.

El VDSL, perteneciente a la familia tecnológica del xDSL, otorga una tasa de transferencia muy elevada. Se trata de una tecnología de acceso a Internet de banda ancha, que transmite los impulsos sobre pares de cobre. El VDSL es una evolución del ADSL (Asymmetric Digital Subscriber Line), y puede suministrarse de manera asimétrica (52 Mbps, Megabits por segundo, de download y 12 Mbps de upload) o de manera simétrica (26 Mbps tanto en subida como en bajada), en condiciones ideales, es decir, sin resistencia de los pares de cobre y con una distancia nula a la central. La tecnología VDSL se sirve de cuatro canales para la transmisión de datos, dos de ellos para descarga y dos para subida. De esta forma se incrementa la potencia de transmisión (ver Nota 2). La norma VDSL adoptada por la Unión Internacional de Telecomunicaciones (UIT) establece una velocidad de transmisión habitual de hasta 50 Mbps en sentido descendente, aunque en general es de cerca de los 23 Mbps y 4 Mbps en sentido ascendente.

Es posible instalar el sistema VDSL partiendo de oficinas centrales o cabinas alimentadas con fibra óptica situadas cerca de los locales del cliente. Pese a su juventud, esta tecnología cuenta con las versiones VDSL1 y VDSL 2. La segunda, aprobada por la UIT en febrero de 2006 (tras un acuerdo en mayo de 2005), utiliza la misma modulación que el ADSL (DMT), puede alcanzar velocidades de 200 Mbps simétricos (tanto de subida como de bajada) y sus capacidades de Quality od Service (QoS) están integradas.

La transmisión de televisión de alta definición por red es la aplicación para la que más se está utilizando la tecnología VSDL, capaz de transmitir vídeo comprimido, una señal en tiempo real poco apta para los parámetros de retransmisión de error 
utilizados en las comunicaciones de datos (ver Nota 3). Los datos hacia el usuario son difundidos a cada equipo de usuario final o transmitidos a un hub desde donde se distribuyen a los usuarios finales mediante multiplexación TDM (Time Division Multiplexing) (ver Nota 4). Una comparativa entre VDSL1 y VDSL2 (Tabla 2) refleja la evolución de la segunda generación.

Tabla 2. Comparativa entre VDSL1 y VDSL2

Parámetro

Ancho de banda

Alcance

Compatibilidad DSL

Ninguna

Calidad de servicio

Ninguna

Eficiencia de transmisión Ninguna

(Trellis, SRA, GCI) (Ver Nota 5)
ADSL, ADSL2, ADSL2+

VDSL2

$30 \mathrm{Mhz}$

$3 \mathrm{~km}$.

Dual Latency, Pre-Emption

Obligatoria

Fuente: Elaboración propia a partir de datos de CECABLE, ZyXEL y Telefónica (2008).

La tecnología VDSL2 aporta un rendimiento mayor que su predecesor para bucle corto, un rendimiento mejorado en cuanto a esquema de eficiencia de transmisión, puede alcanzar al $90 \%$ de clientes por el hecho de permitir una distancia desde la 
central de $3 \mathrm{~km}$. gracias a la calidad de servicio, da acceso sin escollos a los servicios de triple play y permite reutilizar la infraestructura de ADSL existente, diferencia sustancial con relación a la primera generación de VDSL. Además, se combina con tecnologías de fibra óptica, como la FTTB (Fiber to the Building), FTTC (Fiber to the Curb) o FTTN (Fiber to the Node).

Las nuevas redes, cuyo ritmo de despliegue es sostenido, están preparadas para soportar comunicaciones de banda ancha. Su diseño ha sido concebido desde la convergencia tecnológica: se trata de redes de nueva generación dotadas de capacidades multiservicio (una sola red para todas las aplicaciones y que soporta múltiples formas de acceso).

Los servicios de televisión sobre IP, más allá de experiencias piloto como la distribución de televisión sobre IP multicast de 38 canales simultáneos con codificación MPEG-2 a 4 Mbps sobre la propia red de datos (Turró et altri, 2007), dinamizan y se están convirtiendo en un indicador de eficiencia del engranaje de la cadena de valor de la Sociedad de la Información. En la base observamos a los creadores de contenidos (empresas productoras, editoras y discográficas, autores, distribuidores), los mismos contenidos, ya en su mayor parte digitalizados, el broker de contenidos, el proveedor del servicio, el proveedor de red (como el cable, el satélite, soluciones DSL o las diversas plataformas de TV digital), el proveedor de acceso personalizado (cable o DSL) y el proveedor de la plataforma residencial (home platform o videoconsola).

Las opciones de interactividad del VDSL y de los operadores de cable están liderando la evolución de los servicios de televisión (Prado, 2006), que caminan hacia una generación impensable pocos años atrás (Tabla 3). Así, de la escasez de contenidos del modelo analógico se ha saltado de la plétora de canales de audio y 
vídeo de la televisión digital (ver Nota 6), la opción de suscripción a canales a la carta, el pago por visión (PPV) y algunas experiencias de aplicaciones interactivas. Las primeras experiencias piloto de televisión por ADSL, en 2002, añadieron una gran variedad de tipos de contenidos (juegos o composiciones multimedia). Los sistemas ADSL aparecen y se desarrollan a en la década de los 90 del siglo XX. Enfocados al comienzo a la provisión de servicios de televisión y vídeo bajo demanda, y dotados de velocidades que no superaban los 1,5 Mbps, estos sistemas presentaban el valor añadido de reutilizar el par de abonado para proporcionar un flujo digital dedicado a cada abonado (no compartido) y asimétrico. Las velocidades alcanzan hasta los $8 \mathrm{Mbps}$ (sentido red-cliente) y $800 \mathrm{Kbps}$ (sentido cliente-red), lo que permite afrontar los requerimientos de los servicios de vídeo interactivo y de acceso a Internet y otros servicios multimedia.

La generalización de la televisión sobre tecnología DSL estableció una distribución personalizada de contenidos, el acceso a servicios de información multimedia interactivos, la compra, venta o alquiler de contenidos bajo demanda y, acaso lo más rupturista, la interactividad con otros usuarios.

Tabla 3. Evolución de los servicios de televisión de 1990 a 2008

Fase de la TV Cantidad de Canales a la carta Pago por visión Interactividad Canales Elevada (PPV)

$\begin{array}{lllll}\text { TV analógica } & \text { No } & \text { No } & \text { No } & \text { No } \\ \text { TV digital } & \text { Sí } & \text { Sí } & \text { Sí } & \text { Sí }\end{array}$


Planes piloto

Sí

Sí

Sí

No

TV ADSL

TV ADSL

Sí

Sí

Sí

Sí

generalizada

IPTV

Sí

Sí

Sí

Sí

Fuente: Elaboración propia a partir de datos de CECABLE, ZyXEL y Telefónica (2008).

El uso del protocolo IP, cuya regulación es muy incipiente (Urrea, 2007), evita sobremanera la dependencia de sistemas propietarios. Las técnicas de "vídeo digital conmutado" se implementan partiendo de IP multicast, tecnología que permite la comunicación "uno a varios" en redes IP. Cada canal de televisión es transmitido en una dirección IP multicast. Para sintonizar un canal determinado, un descodificador debería suscribirse al grupo multicast correspondiente enviando un paquete IGMP hasta el router de acceso, que hace llegar los paquetes correspondientes a través de la subred por la que se recibió la solicitud (ver Nota 7). En cuanto al vídeo bajo demanda, implica dos canales, uno unidireccional (por el que se recibe el vídeo) y otro bidireccional de control del contenido (a través del cual el cliente transmite los comandos de control). El bidireccional utiliza un protocolo de diálogo con el servidor de vídeo. Pese a la proliferación de protocolos de control propietarios, que requieren una aplicación cliente específica, han surgido estándares como RTSP (Real Time Streaming Protocol), soportados por numerosos servidores de vídeo. Los contenidos de vídeo en tecnologías web utilizan plugins o extensiones de HTML (definidas por el Advanced Television Enhancement Forum) que permiten la mezcla de vídeo y gráficos en una página (ver Nota 8). Las aplicaciones de televisión interactiva se 
implementan como aplicaciones web. En cuanto a la protección de contenidos, las plataformas de televisión digital para entornos abiertos de difusión (redes terrestres o vía satélite) requieren cifrar sus contenidos para restringir el acceso a los no abonados. De los sistemas de protección de contenidos, Digital Video Broadcasting (DVB) acordó estandarizar tan sólo el algoritmo de aleatorización del flujo de datos y establecer la posibilidad de incorporar una interfaz común (CI, Common Interface) en el IRD para que pudieran coexistir diferentes esquemas de acceso condicional simultáneamente.

\section{Aplicaciones prácticas de la televisión mediante VDSL}

La pluralidad tecnológica que envuelve a la televisión (Gueguen, 1996) puede ser aliada o amenaza para las experiencias de TV a través de VDSL. La oportunidad proviene de la mayor tendencia del consumidor a la diversificación tecnológica a la hora de recibir el audiovisual, el nuevo entorno de Multimedia Home Platform (MHP), que, en la encrucijada entre televisor y ordenador, bebe de la fuente digital convergente, la multiplicación de canales y la creciente demanda de servicios interactivos. Los tres perfiles de MHP dependen de la capacidad del receptor. El Enhanced Broadcast Profile, definido en MHP 1.0, se concibe para la descarga (no incluye canal de retorno), a través del canal de broadcast, de aplicaciones con interactividad local (información de entrada enviada desde el mando a distancia, gráficos en la pantalla, selección entre múltiples vídeos o audios); el Interactive Broadcast Profile (MHP 1.0) incluye canal de retorno y permite una comunicación bidireccional con el proveedor de servicios interactivos (aplicaciones como vídeo bajo demanda, comercio electrónico, tele-voto o concursos interactivos); el Internet Acces Profile (MHP 1.1) incluye las capacidades de los anteriores y permite el acceso a Internet (ver Nota 9). 
En contraste, se detecta una masa crítica nada desdeñable ajena aún a los cantos de sirena de ofertas unificadoras como el triple play (Red.es, 2006; Observatorio Nacional de las Telecomunicaciones y la Sociedad de la Información-ONTSI, 2007), cierta desorientación e incluso cierto conservadurismo a la hora de apostar por nuevos tecnologías de las que proceda el servicio televisión, y el hecho una mayor cantidad de canales no implica un aumento en el seguimiento de más cantidad de ellos (Kalinske, 1994).

El MHP, sistema intermediario abierto, diseñado por el proyecto DVB y estandarizado por el European Telecommunications Standards Institute (ETSI) o Instituto Europeo de Normas de Telecomunicaciones, define una plataforma común para las aplicaciones interactivas de la televisión digital, de forma independiente tanto del proveedor de servicios interactivos como del receptor de televisión utilizado. MHP favorece la constitución de un mercado horizontal donde aplicaciones, red de transmisión y terminales MHP pueden ser suministrados por proveedores o fabricantes independientes (ver Nota 10).

Para la generalización de este universo, el establecimiento de redes de banda ancha, pese al esfuerzo inversor que implica (Howard y Carroll, 1993), especialmente las que contienen fibra óptica en la troncal (HFC, Hybrid Fibre Coaxial) o en toda su extensión (FTTH, Fibre-to-the-home y otras variantes), es clave. Los beneficios de la tecnología digital (Castells, 1995 y 1997; Negroponte, 1995) así lo justifican, pese a ciertas posibles disfunciones en la interacción cultural (Ogburn, 1933; Postman, 1993). Sea como fuere, el salto del consolidado triple play (televisión + telefonía + Internet) hacia el nuevo paradigma del quadruple play refleja una tendencia ineludible. En ese contexto, la oferta televisiva debe posicionarse en un mercado muy fragmentado (Smith, 1985; Kopp, 1990; Silj, 1992; Smellie, 1995). El despliegue por continentes del servicio televisivo de VDSL demuestra una correlación favorable a los países cuya 
evolución tecnológica y en clave de Sociedad de la Información y el Conocimiento es más ágil (Tabla 4).

Tabla 4. Despliegue por continentes del servicio de televisión por VDSL en 2008

Europa

Alemania

Bélgica
VDSL (operador que lo ofrece)

Deutsche Telekom: 50 ciudades cuentan con VDSL.

NetCologne: Colonia (VDSL2)

Kabel Deutschland Unitymedia

T-Home

Eslovenia

T.2.net: VDSL desde el 1 de octubre de 2005, con servicio de Internet (velocidades de $1 \mathrm{Mpbs} / 256 \mathrm{kbps}$ a $20 \mathrm{Mbps} / 10 \mathrm{Mbps}$ ), televisión y teléfono en más de 120 ciudades.

España Telefónica: uso incipiente de VDSL en sus nuevas redes de fibra óptica, enfocándola al servicio de TV Imagenio.

Finlandia

OPOY: en Oulu.

Auria: en Turku.

Francia Erenis: Internet y teléfono sobre VDSL2 en París (60 Mbps de bajada y 6 Mbps de subida). 
Holanda KPN: VDSL desde diciembre de 2005 (entre 20 y $30 \mathrm{Mbps}$ ). Mediante VDSL2 llega a 30 Mbps simétricos.

Suecia Bredbandsbolaget AB: VDSL en algunas zonas.

Suiza $\quad$ Swisscom: VDSL desde 2007.

América

Bolivia ENTEL.net: servicio de VDSL en La Paz, Cochabamba, y Santa Cruz (conexión MODEM, LRE y ADSL).

ITS TV por cable: VDSL en Santa Cruz.

Canadá SaskTel: en Saskatchewan, MTS: en Manitoba.

Bell ExpressVu: en Ontario y Québec.

Chile MasNet: en Santiago, Concepción y Valparaíso.

Estados Unidos Qwest: televisión e Internet sobre VDSL en Denver (Colorado), Omaha, Nebraska y Phoenix (Arizona).

México Telmex: VDSL para ofrecer IPTV en diversas zonas.

Asia 
Corea del Sur Numerosos operadores ofrecen VDSL, principal tecnología del mercado.

Hong Kong HGC: en diversos barrios.

Japón NTT, KDDI y otros proveedores (VDSL2 junto con servicios de FTTH).

Taiwán CHT, TFN y NCIC (VDSL2).

Oceanía

Australia TransACT: ofrece televisión digital, Internet y aplicaciones WAN sobre su red FTTC (Fibre-To-The-Curb).

Fuente: Elaboración propia a partir de datos de CECABLE, ADSLZONE.net, Forcis.com, ZyXEL y Telefónica (2008).

Analizando los datos de diversos países, nos hallamos ante una realidad, la de la TV a través de VDSL, pujante. En el caso español, Telefónica utiliza esta tecnología en sus nuevas redes de fibra óptica, enfocándola al servicio de televisión Imagenio (nombre comercial de la televisión digital interactiva del incumbentes), basado en tecnología ADSL2+, que requiere unas condiciones mínimas para su correcto funcionamiento. Así, la velocidad de bajada es de 6016 Kbps y la de subida es de 520 Kbps (ver Nota 11). Imagenio no llega a todos los abonados correctamente, ya que depende de parámetros como la distancia con la central telefónica, el estado del cableado de la línea de teléfono, la red interior del cliente y gran ancho de banda. Imagenio utiliza ADSL y ADSL2+ y tecnología IPTV de transmisión de televisión 
sobre redes IP. El servicio también opera en Chile desde 2007 (bautizado como "On Demand"). La oferta básica consta de 65 canales de televisión y 15 canales de audio con emisión en abierto, además de las modalidades de PPV y vídeo bajo demanda.

La Comisión del Mercado de la Telecomunicaciones (CMT) española se plantea como espíritu regulador del uso de FTTH y VDSL2 la evaluación de la competencia, la determinación de los mercados, el hecho de compartir o no en función de las necesidades de los núcleos territoriales. La clave de la regulación residirá en diferenciar entre áreas competitivas y áreas de no competencia, para que el despliegue de red llegue universalmente, independientemente de la situación comercial que pueda o no existir en la zona. Los modelos americano o francés apuestan por una pléyade de operadores que desplieguen sus redes en las zonas donde consideren que pueden competir (Fondevila Gascón, 2008). La CMT calcula el despliegue de fibra óptica en un plazo de 5 a 10 años, combinado con tecnologías inalámbricas como WiMax y Wifi (ver Nota 12). En caso de que los operadores no inviertan en el despliegue de infraestructura, otro tipo de instituciones (probablemente organismos gubernamentales, ayuntamientos $\mathrm{u}$ otro tipo de entidades privadas) podrían asumir el despliegue y revender el servicio de utilización de la red. Algunos operadores se resistirán a subcontratar las redes a terceros si consideran que pueden ser más rentables en propiedad.

Las ofertas de VDSL de Portal DSL y T-Home en Alemania presentan un relativo grado de madurez. T-Home ofrece Internet, 70 canales de televisión, regala 10 películas al mes y partidos de la liga de fútbol alemana. Extrapolando servicios, la tarifa en España se puede situar en un 37,5\% superior al servicio equivalente en Alemania (ADSLZone, 2008). Otro país cuyo VDSL está bastante desarrollado es Eslovenia, con ofertas de VDSL2 de 40 y $60 \mathrm{Mbps}$ a precios razonables y de fibra óptica hasta el hogar (FTTH) hasta 1 Gbps simétrico. 
En el caso latinoamericano (IDC Latin America, 2007), Argentina, Brasil, Chile y México lideran el mercado de la banda ancha. Bolivia, Chile y México acogen las experiencias primigenias en TV por VDSL. El triple play ya cuenta con adeptos en Argentina, Brasil, Chile, Colombia, El Salvador, Guatemala, Honduras, México, Panamá, Puerto Rico, Venezuela y Uruguay. La televisión por cable es liderada por México, Brasil y Colombia. Los servicios de IPTV (televisión sobre Internet) descuellan en Argentina, Brasil y Chile.

En Francia, cuya banda ancha comercial pivota sobre los 15 Mbps, Issy-lesMoulineaux (departamento de Hauts-de-Seine) acogió en 2005 una prueba experimental de France Telecom con el VDSL. Las velocidades manejadas fluctuaban entre los 40 y los $50 \mathrm{Mbps}$ de descarga utilizando el mismo par de cobre que llegaba hasta el hogar, y 25 Mbps en upload (ver Nota 13). El tiempo de instalación se limitó a un mes. Las implicaciones que puede implicar una extensión del VDSL, pese a quedar siempre lejos de las soluciones recomendables (HFC y, sobre todo FTTH), podrían impulsar la investigación, la intercomunicación y proyectos colaborativos, una televisión híper personalizada (The Television Me, parafraseando al The Daily Me de Negroponte) cuya vertiente publicitaria disparará los ingresos para televisiones de ámbito local, amén de impulsar aplicaciones como las videotecas en línea o compras desde cualquier dispositivo digital. La posibilidad de emitir un canal de televisión desde casa ya no sería una quimera (ver Nota 14), así como el vídeo bajo demanda, la distribución de canales en alta definición MPEG-4 (ver Nota 15) y la transmisión de datos de empresa en red simétrica. Las pruebas de ADSL2 en el Reino Unido han llevado hasta los $18 \mathrm{Mbps}$ (los servicios de streaming pueden ofrecer calidad DVD).

La realidad tecnológica asiática cabalga a un ritmo intenso, como lo demuestran los escarceos con los nuevos estándares DSL de Corea del Sur, Hong Kong, Japón y 
Taiwán. En Japón, el objetivo para 2010 consiste en alcanzar el 60\% de penetración de banda ancha en un país acostumbrado a redes de fibra óptica de 100 Mbps y los consiguientes servicios avanzados de radio y televisión bajo demanda.

La vitalidad de los proyectos de IPTV está moviendo a los principales actores del sector. Una muestras de ello es el acuerdo a finales de 2008 por el que Alcatel-Lucent debe suministrar al proveedor de energía eléctrica EPB una solución de televisión sobre IP por un importe de 45,5 millones de euros, lo que permitirá a la compañía estadounidense ofrecer servicios avanzados de televisión y comunicaciones en Chattanooga Tennessee. El despliegue de la red de fibra óptica se llevará en un plazo estimado de cinco años. El servicio se basa en la arquitectura de distribución de servicios triple play TPSDA (Triple Play Service Delivery Architecture) de AlcatelLucent, que potencia la entrega de servicios de televisión de EPB. Alcatel-Lucent asume la solución de extremo a extremo, incluida la cabecera de vídeo, la infraestructura de la red, la plataforma del servicio de TV IP, los sistemas de operación OSS, el diseño de la arquitectura, las pruebas, la integración, el despliegue, la operación y el mantenimiento. La solución permite ofrecer servicios avanzados de TV, incluido vídeo bajo demanda (VoD), con contenidos de alta definición (HD).

Acciones que el periodismo audiovisual del presente y aún más del futuro debe proporcionar cómodamente a la audiencia, como descargar vídeos de Internet en fracciones de segundo, descargarse películas completas de DVD en pocos minutos o transmitir una videoconferencia sin retardos precisan de una conexión a Internet que debe rondar velocidades de $100 \mathrm{Mbps}$. Algunos operadores telecomunicación de Japón, Suecia, Corea, Francia y Finlandia ya ofrecen comercialmente estos anchos de banda. El crecimiento del ancho de banda transpacífico (PriMetrica, 2008) y las previsiones que a partir de 2008 la demanda se duplique cada dos años a raíz de los 
cuello de botella provocados por la IPTV (IDC-Tellabs, 2008) reflejan la necesidad de autopistas de la información de más caudal.

En España, la primera oferta a 100 Mbps llegaría a los hogares a finales de 2008 de la mano de Ono. La prueba piloto se llevaría a cabo en el área metropolitana de Valladolid gracias a la tecnología Docsis 3.0, que mejora la capacidad y el rendimiento de las redes de cable de última generación. El objetivo era probar esta tecnología durante seis meses para poder ofrecer el servicio comercialmente en el último trimestre del año en Madrid, Valencia y Barcelona. Para ello, la operadora tenía previsto llevar a cabo un despliegue masivo de esa tecnología gracias a una inversión de 75 millones de euros que le permitiría completar la nueva red a lo largo de 2009. Ono no aclaró si el Internet ultrarrápido tendría un megaprecio, porque en foros de internautas se especulaba con 100 euros al mes.

Ono, cuya red llegaba a 6,9 millones de hogares, sería el primer operador en disponer de una oferta con carácter estatal de $100 \mathrm{Mbps}$, pero no el primero que lo ofreciera en España. La compañía Adamo, de origen sueco, ya comercializaba 100 Mbps en algunos pueblos de Asturias. En el conjunto de España, el tramo de velocidad más habitual fluctuaba entre los 2 y los 9,9 Mbps, que contrataba el 72\% de los ocho millones de abonados.

Telefónica, que controlaba más de la mitad del mercado de banda ancha en España, estaba llevando a cabo pruebas piloto en Madrid y Barcelona con velocidades de 30 Mbps. Según la regulación final propuesta por la CMT, podían peligrar los $30 \mathrm{Mbps}$ (que podían ofrecerse mediante tecnología VDSL2) y la propia red de fibra óptica hasta el hogar, la única capaz de ofrecer velocidades de 100 Mbps o superiores. 
Las aplicaciones en VDSL deben partir de una cauterización en España de la brecha digital respecto a los países más desarrollados, ya que los accesos a Internet crecieron en 2007, pero menor medida que en la mayoría de los Estados desarrollados (OECD, 2008) (ver Nota 16). La banda ancha era más cara que la media en relación con la renta per cápita. El acceso a Internet supone casi el 3\% de los ingresos de los usuarios. La penetración de la banda ancha en España era de 18,04 líneas por cada 100 habitantes a finales de 2007, casi dos puntos por debajo de la media de los países de la OCDE $(19,96)$. En los países más industrializados habían crecido los accesos a Internet a un mayor ritmo que en España. Los planes del Gobierno (Ingenio 2010, Avanza e Internet Rural) y la multiplicación de la competencia a Telefónica no impidieron el relativo retroceso de España. En 2002, la penetración de banda ancha era de poco más del $2 \%$, pero la diferencia con la media de la OCDE era de 1,86 puntos.

España era el octavo país de la OCDE donde la banda ancha era más exigente para la economía doméstica. Sólo México, Turquía, Eslovaquia, República Checa, Polonia, Hungría y Portugal están empeoraban la situación. Los países que mejores precios presentan respecto a su renta per cápita eran Luxemburgo, Suiza, Finlandia, Dinamarca, Reino Unido, Irlanda y Alemania.

Respecto a los precios absolutos, el informe revelaba una situación correcta en las ofertas de más bajo precio, con una media de 21,96 dólares mensuales (14 euros) en la OCDE frente a los 20,9 dólares de España (13,3 euros), pero la media del abono mensual de las ofertas más caras (las referidas generalmente a empresas) era de 242,99 dólares (154 euros) en España, casi el doble que el de la OCDE.

En cuanto a la calidad del servicio que prestaban las operadoras españolas, la media de la velocidad máxima de acceso a Internet españolas por ADSL era de 20,4 Mbps, 
frente a los 22,3 Mbps de la OCDE. Los países más avanzados eran Corea, Japón, Reino Unido y Suecia. Sólo 11 países disponían de ofertas comerciales de acceso a Internet a través de fibra óptica (FTTH). La más poderosa era Japón (1 Gbps), y Suecia, Corea, Francia, Finlandia ofrecían 100 Mbps. En tres años se había cuadruplicado la velocidad media de acceso que se ofertaba en los países de la OCDE (desde los menos de 2 Mbps de finales de 2004 hasta los casi 9 Mbps de 2007). Excluyendo a Japón, la media de máximas velocidades ofertadas por los ex monopolios de los países de la OCDE era de 26,5 Mbps, mientras que la de Telefónica era de 10,2 Mbps.

Los suscriptores a banda ancha en la OCDE eran 235 millones en diciembre de 2007, un 18\% más sobre los 200 millones de suscriptores un año atrás. Dinamarca, Holanda, Islandia, Noruega, Suiza, Finlandia, Corea y Suecia lideraban la OCDE en términos de penetración Las conexiones de Fibre-to-the-home (FTTH) y Fibre-to-thebuilding (FTTB) comprendían el 8\% de todas las conexiones de banda ancha en la OCDE. Las conexiones en fibra llegaban al $40 \%$ de las suscripciones japonesas y al $34 \%$ en Corea. Estados Unidos se confirmaba como el mayor mercado de banda ancha en la OCDE, con 69,9 millones de suscriptores (el 30\% del total de conexiones de banda ancha en la OCDE).

\section{Conclusiones}

La IPTV a través de VDSL reforzará el papel de la interactividad del medio y supondrá un nuevo impulso para el periodismo audiovisual siempre que la red de banda ancha de fibra óptica se extienda y conceda los caudales suficientes como para garantizar la calidad del servicio, como mínimo en el contexto bajo demanda. El análisis de las incipientes experiencias de televisión a través de VDSL en diversos países permite establecer una prospectiva que afecta a las diversas aplicaciones 
audiovisuales. El servicio de televisión adquiere así protagonismo ante los de telefonía e Internet dentro de los servicios de triple play, y puede combinarse en movilidad con el quadruple play extendiéndose a otros soportes que la exponencialidad del desarrollo tecnológico invita a imaginar. Las tendencias y recomendaciones para el audiovisual del paradigma IP son éstas:

-Es perceptible la combinación armoniosa de una infraestructura tecnológica consistente con contenidos atractivos. Así, la misión reside en ofrecer al espectador contenidos de calidad muy elevada. Para ello hay que utilizar redes de banda ancha y accesos de muy alta velocidad $\mathrm{y}$, a poder ser, garantizados.

-Los operadores de telecomunicación por cable y los MSO (Multiple Service Operator) y, en general, los operadores que renuncian al acceso indirecto en beneficio de disponer de red propia, apuestan por despliegues que permitan ofrecer IPTV. Estas redes se están centrando en su confiabilidad, seguridad y escalabilidad para acoger con garantías contenidos audiovisuales cuyo peso en bits por segundo sea elevado. De hecho, el objetivo final puede consistir en establecer unos acuerdos de garantía de servicio ad hoc (microSLAs, microService Level Agreements), en función de necesidades puntuales del usuario, para que la red no se convierta en una commodity y permita retornos de la inversión para sus propietarios a largo plazo o sine die. De hecho, entre los requisitos de IPTV se hallan el tiempo real y un ancho de banda exclusivo, lo que abona estas estrategias rentabilizadoras.

-La pantalla de televisión debe configurarse como autosuficiente en un entorno multiplataforma y multiterminal como el actual. Así, los operadores de telecomunicaciones y los gestores de canales deben ofrecer servicios interactivos de información sin que necesariamente precisen de acceso a Internet y PC como 
terminal de cliente. Los contenidos interactivos deben concebirse con un acceso sencillo e intuitivo desde la televisión del hogar.

-La multiplicación de ofertas televisivas puede actuar como freno de las experiencias de IPTV, de la misma manera que ocurriera en su momento con las plataformas digitales vía satélite e incluso con los operadores de cable cuando se modificó la legislación de 1995. En cambio, el crecimiento sostenido de accesos a Internet y el advenimiento de generaciones más digitalizadas genera una masa crítica que puede modificar tendencias y sorprender a los propios emprendedores del sector, igual que ocurrió con el fenómeno de los SMS en telefonía móvil en España.

\section{Bibliografía}

ADSLZONE.NET, Informe Diferencias entre el VDSL de Alemania y el que llegará a España de manos de Telefónica, ADSLZONE.NET, Madrid, 2008.

BECERRA, Martín, Sociedad de la información: proyecto, convergencia, divergencia, Editorial Norma, Buenos Aires, 2003.

CASTELLS, Manuel, La ciudad informacional: tecnologías de la información, reestructuración económica y el proceso urbano-regional, Alianza, Madrid, 1995.

CASTELLS, Manuel, La Era de la Información, Alianza, Madrid, 1997.

COMISIÓN EUROPEA, Europa y la sociedad global de la información (Informe Bangemann), Recomendaciones al Consejo Europeo, Comisión Europea, Bruselas, 1994. 
COMISIÓN EUROPEA, Libro Verde Sobre la convergencia de las telecomunicaciones, los medios de comunicación y las tecnologías de la información y sobre sus consecuencias para la reglamentación en la perspectiva de la Sociedad de la Información, Comisión Europea, Bruselas, 1997.

DELGADO, Matilde; FERNÁNDEZ QUIJADA, David. "IPTV: estructura de mercado y tipología de la oferta en España", ZER, 22, pp. 413-428, 2007.

ENTER-IDATE, DigiWorld España 2007, Enter (Centro del IE Business School para el Análisis de la Sociedad de la Información y las Telecomunicaciones), Madrid, 2007.

FONDEVILA GASCÓN, Joan Francesc, El cable y la Banda Ancha en España, 2007, CECABLE, Terrassa, 2008.

GARNHAM, Nicholas, "Le développement du multimedia: un déplacement des rapports de force", en AA.VV., La Société face au Multimedia, IDATE, Montpellier, pp. 153-174, 1996.

GILDER, George, Telecosm, The World After Bandwidth Abundance, Touchstone, New York, 2002.

GUEGUEN, Claude, "Une société de l'information par la technologie: entre inquiétude et espoir", Communication et langages, $\mathrm{n}^{\circ} 107$, primer trimestre, pp. 33$46,1996$.

HOWARD, Herbert H.; CARROLL, Sidney L., "Economics of the Cable Industry", en A. Alexander; J. Owers; R. Carveth (eds.): Media Economics. Theory and Practice. New Jersey, Lawrence Erlbaum Associates, pp. 245-266, 1993. 
IDC Latin America, Mercado de Servicios de Telecomunicaciones en Latinoamérica, IDC América Latina, Miami, 2007.

IDC-Tellabs, Survey about bandwidth demands, Tellabs Naperville (USA), 2008.

KALINSKE, Tom, "History Repeats Itself: Consumer's Technology and Entertainment". CTMA Quarterly Journal 2, nº 4, 1994.

KOPP, P. (1990): Télévisions en concurrence, París, Anthropos.

MIÈGE, Bernard (1997): La société conquise par la communication II: La communication entre l'industrie et l'espace public, Grenoble, Presses Universitaires de Grenoble.

MILLA, Héctor. "VDSL, red de proximidad y ¿para qué?". Blog de Héctor MillaInnovación audiovisual y televisión 2.0. 31-III-2005.

http:// www.hectormilla.com/article/148/vdsl-red-de-proximidad-y-para-que

NEGROPONTE, Nicholas, Being digital, Knopf, New York, 1995.

ONTSI (Observatorio Nacional de las Telecomunicaciones y la Sociedad de la Información), La Sociedad en Red 2007. Informe Anual, Red.es, Madrid, 2007.

OECD (Organisation for Economic Co-operation and Development), Broadband Growth and Policies in OECD Countries, OECD Ministerial Meeting on the Future of the Internet Economy. Seoul, 2008. 
OGBURN, William, Living with machines, American Library Association, Chicago, 1933.

PESTANO RODRÍGUEZ, José Manuel, Las tecnologías de la información y la comunicación en la sociedad actual: una aproximación analítica, Tauro Producciones, Tegueste, 2001.

POSTMAN, Neil, Technopoly. The surrender of Culture to Technology, Alfred A. Knopf, New York, 1993.

PRADO, E. et al., Televisió interactiva. Simbiosi tecnològica i sistemes d'interacció amb la televisió, Consell de l'Audiovisual de Catalunya, Barcelona, 2006.

PRIMETRICA, INC., Informe TeleGeography's Global Internet Geography, TeleGeography Research, Washington, 2008.

READING, Viviane, Europe on the way to a high speed Internet economy, Launch Press EITO, Brussels, 2008.

RED.ES, Análisis de la oferta de los operadores de cable, Red.es, Madrid (Observatorio de las Telecomunicaciones y de la Sociedad de la Información), 2006.

RITZER, George, The Coming of Post-Industrial Society (segunda edición), McGrawHill, New York, 2007.

SILJ, Alessandro, The New Television in Europe, John Libbey, London, 1992.

SMELLIE, Alastair, European Pay TV, Lehman Brothers, London, 1995. 
SMITH, Marvin, Radio, TV and Cable: A Telecommunications Approach, Holt, Rinehart and Winston, New York, 1985.

TURRÓ, Carlos, et al., "Usando intensivamente la red: 152 Mbps de multicast para distribuir TDT, satélite y canales educativos internos en la UPV" (Área de Sistemas de Información y Comunicaciones Universidad Politécnica de Valencia), Boletín de RedIRIS, no 78-79, diciembre 2006-enero 2007.

http://www.rediris.es/rediris/boletin/78-79/ ponencia13.pdf

URREA, Héctor, "Retos regulatorios de la televisión sobre el protocolo IP", Revista AHCIET: revista de telecomunicaciones, Asociación Iberoamericana de Centros de Investigación y Empresas de Telecomunicaciones, Madrid, nº 110, 2007.

\section{Notas}

Nota 1. La consolidación del concepto de triple play ha precedido a la aparición del de quadruple play, que implica, en un entorno de movilidad y conectividad totales (always on), el posible acceso a la red y a sus contenidos en cualquier momento y desde cualquier lugar.

Nota 2. La técnica estándar de modulación puede ser QAM/CAP (Carrierless Amplitude Phase) o DMT (Discrete Multitone Modulation), incompatibles pero cuyo rendimiento es similar. La más habitual es DMT.

Nota 3. Con el objetivo de lograr tasas de error compatibles con el vídeo comprimido, VDSL incorpora un Forward Error Correction (FEC) con suficiente intercalado para corregir todos los errores creados por la aparición de ruidos impulsivos de una especificada duración. 
Nota 4. La multiplexación hacia el usuario presenta otras implicaciones. Los sistemas que utilizan una NT (Network Termination) pasiva deben insertar los datos en un medio compartido, mediante alguna variante TDMA (Time Division Multiple Access) o de FDM (Frecuency Division Multiplexing).

Nota 5. La modulación Trellis (también conocida como Trellis Coded Modulation, o simplemente TCM) es un esquema de modulación que aporta transmisión de información de alta eficiencia sobre canales con ancho de banda limitado, especialmente en telefonía.

Nota 6. La televisión digital aporta mayor calidad de imagen y sonido. La transmisión digital mantiene la calidad extremo a extremo y permite obtener mayor aprovechamiento de los medios de transmisión, aumentando su capacidad en un factor multiplicativo entre 6 y 16. Además, permite nuevos servicios basados en nuevas modalidades de acceso (vídeo bajo demanda, guía electrónica de programación, pago por visión), la incorporación de servicios interactivos sincronizados con las señales de TV y la integración de servicios de vídeo, audio y datos, amén de la transmisión de canales de audio digital. Es susceptible de incorporar en el futuro señales de TV de alta definición.

Nota 7. El transporte de vídeo sobre IP se está estandarizando en el marco de la organización internacional DVB (comité DVB-IPI), que en 2003 publicó su primera especificación. Las principales cuestiones de seguridad a tratar son la suplantación de identidad (spoofing), los ataques de denegación de servicio (DoS) y el robo del propio servicio. Soluciones como las Validaciones de Nodos de Red (Network Node Validations) se revelan muy útiles. 
Nota 8. En esta tecnología se emplean URLs o etiquetas adicionales para implementar operaciones diversas (cambio de canal de TV o solicitud de un contenido de vídeo bajo demanda). Se utilizan protocolos de recepción de datos por un canal unidireccional como UHTTP (Unidirectional HTTP). Se emplean extensiones de HTML para la sincronización de contenidos de TV con la recepción de datos.

Nota 9. La española ADNStream (www.adnstream.tv) dispone de diversas opciones de modelos avanzados de televisión, comenzando por un prototipo de TV que absorbe un PC y se comporta como un híbrido. Este modelo ha sido desarrollado por la empresa Sincroniza. Se trata de un televisor con media center incorporado que permite acceso a todo tipo de contenido en audio y vídeo. Funciona en Windows y Linux, y permite visionar un programa y chatear o revisar el correo electrónico. La otra plataforma de la empresa española es Zappinternet (www.zappinternet.com). ADNStream incluye un servicio VOD (Vídeo Bajo Demanda) basado en pagos por SMS para acceder a un film durante 24 horas de plazo.

Nota 10. El estándar MHP soporta una tipología diversa de aplicaciones interactivas: Guía Electrónica de Programas (EPG), servicios de información como noticias, deportes o superteletexto, aplicaciones sincronizadas con el contenido de los programas, e-mail e Internet y otros servicios como comercio electrónico, servicios de educación o salud, dentro de las aplicaciones del Estado del Bienestar propias de la Sociedad de la Información y el Conocimiento (Comisión Europea, 1994 y 1997).

Nota 11. La máxima atenuación de bajada en Imagenio es de 40 dB, y la de subida, de $21 \mathrm{~dB}$. El margen de ruido de bajada/subida es de $9 \mathrm{~dB}$. 
Nota 12. España cuenta en 2008 con 350.000 líneas con nodos de fibra óptica. El objetivo es que existan 492 centrales con capacidad para ofrecer fibra óptica hasta el hogar (FTTH), fibra óptica hasta el edificio (FTTx ) y VDSL2.

Nota 13. El VDSL se instala en unos armarios, en plena calle, conectados a las centrales telefónicas. La zonificación correspondiente afecta a un número de usuarios que oscila entre los 100 y los 2000. Los armarios se hallan cerca del abonado y de la central telefónica. El test estaba autorizado por la entidad reguladora en Francia, el ART.

Nota 14. El servicio de televisión de Telefónica Imagenio requiere un ancho de banda de unos 4 Mbps de bajada, y la TVHD (Televisión de Alta Definición) unos 15 Mbps de emisión. Los 25 Mbps de subida conceden opciones sugerentes.

Nota 15. Moving Picture Experts Group (Grupo de Expertos de Imágenes en Movimiento), conocido como MPEG, es un grupo de trabajo del ISO/IEC (Organización Internacional para la Estandarización/ Comisión Electrotécnica Internacional) encargado de desarrollar estándares de codificación de audio y vídeo. MPEG-4 expande MPEG-1 para soportar "objetos" audio/vídeo, contenido 3D, codificación de baja velocidad binaria y soporte para gestión de derechos digitales (protección de copyright).

Nota 16. http://www.oecd.org/sti/ict/broadband. 